SUNY-NTG-94/6

\title{
Spectral sum rules and Selberg's integral formula
}

\author{
Jacobus Verbaarschot \\ Department of Physics \\ SUNY, Stony Brook, New York 11794
}

\begin{abstract}
Using Selberg's integral formula we derive all Leutwyler-Smilga type sum rules for one and two flavors, and for each of the three chiral random matrix ensembles. In agreement with arguments from effective field theory, all sum rules for $N_{f}=1$ coincide for the three ensembles. The connection between spectral correlations and the low-energy effective partition function is discussed.
\end{abstract}

SUNY-NTG-94/6

January 1994 


\section{Introduction}

As has been shown by Leutwyler and Smilga [1], chiral perturbation theory of the low-energy effective QCD partition function leads to sum rules for the inverse powers of the eigenvalues of the Dirac operator in QCD. On the other hand these sum rules can also be derived from chiral random matrix theory [2, 3] with only the symmetries of the QCD Dirac operator as input. They follow from the microscopic correlations between the eigenvalues near zero virtuality, which are conjectured to be universal [2, 4]. In [3] we have argued that there are three different universality classes: $S U(2)$-gauge theory with fundamental fermions, $S U\left(N_{c}\right), N_{c} \geq 3$ with fundamental fermions and $S U\left(N_{c}\right)$, $N_{c} \geq 2$ with adjoint fermions. The Dirac operator can be chosen real, is complex or can be regrouped into quaternions, respectively. In analogy with the classical random matrix ensembles [6], the corresponding chiral random matrix theory will be called the chiral orthogonal ensemble (chGOE), the chiral unitary ensemble (chGUE) and the chiral symplectic ensemble (chGSE). As was shown in [3] the spectral correlations of chiral random matrix theory are different in each of the three cases. Indeed, for two or more flavors the effective theory is also different [1, 5]. However, for one flavor, the low-energy effective partition function, with proper identification of the parameters, is the same in each of the three cases [1], and therefore also the spectral sum rules are the same. This leads to the paradox [7] of how an infinite family of sum rules can be the same, whereas the spectrum near zero virtuality is different. This issue will be resolved below by an explicit calculation of all sum rules.

In general, spectral correlation functions can be calculated exactly, but the integrals for the sum-rules can be evaluated analytically only for the simplest correlation functions [4]. However, there is a more direct way to obtain the same results. The spectral sum rules can be expressed in terms of the joint eigenvalue density. The resulting integrals are very complicated, but fortunately, exactly these types of integrals have been considered before by Selberg [8] and can easily be generalized with the help of Aomoto's proof [9] thereof (see [10] for a detailed discussion). Using this technique we are able to derive all sum rules for one and two flavors. 


\section{Leutwyler-Smilga spectral sum rules}

For $N_{f}$ flavors with masses $m_{f}\left(m_{f} \rightarrow 0\right)$ the QCD partition function in the sector with $\nu$ zero modes is defined by

$$
Z_{\nu}^{\mathrm{QCD}}(m)=\left\langle\prod_{f=1}^{N_{f}} \prod_{\lambda_{n}>0}\left(\lambda_{n}^{2}+m_{f}^{2}\right) m_{f}^{\nu}\right\rangle_{S_{\nu}(A)}
$$

where the average is over gauge field configurations with $\nu$ fermionic zero modes weighted by the gauge field action $S_{\nu}(A)$. The product is over all eigenvalues of the Dirac operator. For fermions in the adjoint representation, the doubly degenerate eigenvalues count only once (Majorana fermions) [1]. All sum rules that can be obtained from chiral perturbation theory follow by equating the expansion in powers of $m^{2}$ of the QCD partition function (1) and the low-energy effective partition function. For $N_{f}=1$, the fermion determinant can be expanded as

$$
\prod_{\lambda_{n}>0}\left(\lambda_{n}^{2}+m^{2}\right)=\prod_{\lambda_{n}>0} \lambda_{n}^{2}\left(1+\sum_{p=1} m^{2 p} \sum_{n_{1} \neq \cdots \neq n_{p}} \frac{1}{\lambda_{n_{1}}^{2} \cdots \lambda_{n_{p}}^{2}}\right) .
$$

Therefore, one finds sum rules for the following quantities

$$
S_{p} \equiv\left\langle\sum_{n_{1} \neq 2 \neq \cdots \neq n_{p}} \frac{1}{\lambda_{n_{1}}^{2} \cdots \lambda_{n_{p}}^{2}}\right\rangle
$$

where the average, which includes the fermion determinant in the chiral limit, is with respect to the QCD partition function. For $N_{f}$ flavors, it is clear that sum rules for the quantities

$$
\left\langle\sum_{\text {all } \lambda_{k} \text { different }} \frac{1}{\lambda_{\alpha_{1}}^{2 N_{f}} \cdots \lambda_{\alpha_{n_{N}}}^{2 N_{f}} \lambda_{\beta_{1}}^{2 N_{f}-2} \cdots \lambda_{\beta_{n_{N_{f}}-1}}^{2 N_{f}-2} \cdots \lambda_{\omega_{1}}^{2} \cdots \lambda_{\omega_{n_{1}}}^{2}}\right\rangle .
$$

follow from a similar expansion of the product

$$
\prod_{\lambda_{n}>0} \prod_{f=1}^{N_{f}}\left(\lambda_{n}^{2}+m_{f}^{2}\right)
$$

Notice that in the thermodynamic limit these sum rules are only sensitive to the spectrum near zero virtuality. In other words, they reflect on the microscopic correlations of the spectrum, i.e., correlations between eigenvalues near zero on the scale $1 / V_{4}$. 


\section{Random matrix theory}

In this paper we calculate the sum rules, not via the chiral perturbation expansion of the effective field theory but rather from chiral random matrix theory, which, invoking universality arguments [2, 11], describes the spectrum of the QCD Dirac operator near zero virtuality. In this case the partition function is defined by

$$
Z_{\beta, \nu}(m)=\int \mathcal{D} T P_{\beta}(T) \prod_{f}^{N_{f}} \operatorname{det}\left(\begin{array}{cc}
m_{f} & i T \\
i T^{\dagger} & m_{f}
\end{array}\right)
$$

where $T$ has the symmetries of the corresponding Dirac operator and the masses are in the chiral limit $\left(m_{f} \rightarrow 0\right)$. As discussed above, depending on the universality class the matrix $T$ is real $(\beta=1, \operatorname{chGOE})$, complex $(\beta=2$, chGUE) or quaternion real $(\beta=4$, chGSE). In the latter case the square root of the fermion determinant appears in (6). The matrix $T$ is a rectangular $n \times m$ matrix with $|n-m|=\nu$ (for definiteness we take $m>n)$, so that the 'Dirac operator' in (6) has exactly $\nu$ zero modes. The function $P(T)$ is chosen gaussian:

$$
P_{\beta}(T)=\exp \left(-\frac{\Sigma^{2} \beta n}{2} \sum_{k=1}^{n} \lambda_{k}^{2}\right) .
$$

In this normalization, the chiral condensate equals $\Sigma$ in each of the three random matrix ensembles. The total number of modes is $N \equiv 2 n$. The latter quantity is identified with the volume of space time. The joint probability density of the eigenvalues of the Dirac operator is obtained by transforming to integration variables in which $T$ is diagonal. For $N_{f}$ flavors and topological charge $\nu$ it is given by

$$
\rho_{\beta}\left(\lambda_{1}, \cdots, \lambda_{n}\right)=C_{\beta, n} \prod_{k, l}\left|\lambda_{k}^{2}-\lambda_{l}^{2}\right|^{\beta} \prod_{k} \lambda_{k}^{2 N_{f}+\beta \nu+\beta-1} \exp \left(-\frac{n \beta \Sigma^{2}}{2} \sum_{k} \lambda_{k}^{2}\right),
$$

where $C_{\beta, n}$ are normalization constants.

The expectation values (4) are calculated with respect to the joint probability density (8). To evaluate the integrals, it is convenient to introduce new integration variables

$$
\mu_{k}=\frac{\beta n \Sigma^{2}}{2} \lambda_{k}^{2}
$$

resulting in the joint spectral density (up to a constant)

$$
\rho_{\beta, a}\left(\mu_{1}, \cdots, \mu_{n}\right) d \mu_{1} \cdots d \mu_{n}=\prod_{k<l}\left|\mu_{k}-\mu_{l}\right|^{\beta} \prod_{k} \mu_{k}^{a} \exp \left(-\sum_{k} \mu_{k}\right) d \mu_{1} \cdots d \mu_{n}
$$


where

$$
a=\frac{\left(2 N_{f}-2+\beta+\beta \nu\right)}{2}
$$

Note that for $N_{f}=1$ we have the following remarkable property

$$
\rho_{\beta, a}\left(\beta \mu_{1}, \cdots, \beta \mu_{n}\right) \sim\left[\rho_{\beta=1, a}\left(\mu_{1}, \cdots, \mu_{n}\right)\right]^{\beta}
$$

\section{Recursion relations for Selberg's integral}

The evaluation of the spectral sum rules can be reduced to the calculation of moments

$$
\left\langle\mu_{1}^{p_{1}} \cdots \mu_{n}^{p_{n}}\right\rangle_{\rho_{\beta, a}}
$$

Because all integration variables occur symmetrically, they only depend on the partitioning of the powers and will be denoted by

$$
H_{\beta, a}\left(n_{p}, n_{p-1}, \cdots, n_{0}\right), \quad\left(\sum_{k} n_{k}=n\right)
$$

Here, $n_{k}$ is the number of times the power $k$ occurs in (13). By integrating $\partial_{\mu_{1}} \rho_{\beta, a}\left(\mu_{1}, \cdots, \mu_{n}\right)$ we find the recursion relation

$$
\begin{aligned}
H_{\beta, a}\left(n_{p}, n_{p-1}, \cdots, n_{0}\right) & =(p+a) H_{\beta, a}\left(n_{p}-1, n_{p-1}+1, \cdots n_{0}\right) \\
& +\beta \sum_{k}\left\langle\frac{\prod_{i=1}^{n_{p}} \mu_{i}^{p} \prod_{j=1}^{n_{p}} \mu_{j}^{p-1} \cdots}{\mu_{1}-\mu_{k}}\right\rangle_{\rho_{\beta, a}}
\end{aligned}
$$

The last term can be evaluated by exploiting that the the joint probability density is a symmetric function with respect to all variables. If we denote $\int d x_{1} d x_{2} S\left(x_{1}, x_{2}\right) F\left(x_{1}, x_{2}\right)$ by $\langle F\rangle_{S}$, we can derive for a symmetric function $S\left(x_{1}, x_{2}\right)$ and even values of $q$ the relations

$$
\left\langle\frac{x_{1}^{p} x_{2}^{p-q}}{x_{1}-x_{2}}\right\rangle_{S}=\sum_{l=1}^{q / 2}\left\langle x_{1}^{p-l} x_{2}^{p-q+l-1}\right\rangle_{S}
$$

where we have used that $\left\langle x_{1}^{k} x_{2}^{k} /\left(x_{1}-x_{2}\right)\right\rangle_{S}=0$. For odd values of $q$ a similar relation can be obtained

$$
\left\langle\frac{x_{1}^{p} x_{2}^{p-q}}{x_{1}-x_{2}}\right\rangle_{S}=\frac{1}{2}\left\langle x_{1}^{p-(q+1) / 2} x_{2}^{p-(q+1) / 2}\right\rangle_{S}+\sum_{l=1}^{(q-1) / 2}\left\langle x_{1}^{p-l} x_{2}^{p-q+l-1}\right\rangle_{S} .
$$


In this case the series terminates because

$$
\left\langle\frac{x_{1}^{k+1} x_{2}^{k}}{x_{1}-x_{2}}\right\rangle_{S}=\frac{1}{2}\left\langle x_{1}^{k} x_{2}^{k}\right\rangle_{S} .
$$

With the help of these relations all terms in the sum of (15) can be expressed in the functions $H_{\beta, a}$. As a result we obtain the recursion relation

$$
\begin{aligned}
H_{\beta, a}\left(n_{p}, n_{p-1}, \cdots, n_{0}\right) & =\left(p+a+\frac{\beta}{2} n_{p-1}+\beta \sum_{q=2}^{p} n_{p-q}\right) H_{\beta, a}\left(n_{p}-1, n_{p-1}+1\right) \\
& +\frac{\beta}{2} \sum_{k=1}^{[(p-1) / 2]} n_{p-(2 k+1)} H_{\beta, a}\left(n_{p}-1, n_{p-k-1}+2, n_{p-(2 k+1)}-1\right) \\
& +\beta \sum_{q=4}^{p} n_{p-q} \sum_{l=2}^{[q / 2]} H_{\beta, a}\left(n_{p}-1, n_{p-l}+1, n_{p-q+l-1}+1, n_{p-q}-1\right),
\end{aligned}
$$

where the largest integer smaller than $x$ is denoted by $[x]$, and we used the notation that all arguments of $H$ not shown explicitly are the same as in the l.h.s. of the equation. In general, this recursion relation is very complicated and an analytical solution seems illusive. However for $p=1,2$ a linear recursion relation is obtained which can be worked out analytically. For $p=1$ the result is [10]

$$
\begin{aligned}
H_{\beta, a}\left(n_{1}, n_{0}\right) & =\left(a+1+\frac{\beta}{2} n_{0}\right) H_{\beta, a}\left(n_{1}-1, n_{0}\right) \\
& =\prod_{k=0}^{n_{1}-1}\left(a+1+\frac{\beta}{2}\left(n_{0}+k\right)\right) H_{\beta, a}\left(0, n_{0}+n_{1}\right) .
\end{aligned}
$$

For $p=2$, the result is only slightly more complicated [10:

$$
\begin{aligned}
H_{\beta, a}\left(n_{2}, n_{1}, n_{0}\right) & =\left(a+2+\frac{\beta}{2} n_{1}+\beta n_{0}\right) H_{\beta, a}\left(n_{2}-1, n_{1}+1, n_{0}\right) \\
& =\prod_{k=0}^{n_{2}-1}\left(a+2+\frac{\beta}{2}\left(n_{1}+k\right)+\beta n_{0}\right) H_{\beta, a}\left(0, n_{1}+n_{2}, n_{0}\right) \\
& =\prod_{k=0}^{n_{2}-1}\left(a+2+\frac{\beta}{2}\left(n_{1}+k\right)+\beta n_{0}\right) \\
& \times \prod_{l=0}^{n_{1}+n_{2}-1}\left(a+1+\frac{\beta}{2}\left(n_{0}+l\right)\right) H_{\beta, a}\left(0,0, n_{0}+n_{1}+n_{2}\right) .
\end{aligned}
$$

To obtain the last equality we have used the relation for $p=1$. 


\section{Results for the spectral sum rules}

It is now straightforward to evaluate all sum rules for one and two flavors. First, we consider the case $N_{f}=1$. Since all eigenvalues occur symmetrically in (3) we have

$$
S_{p}=\left(\begin{array}{c}
n \\
p
\end{array}\right)\left\langle\frac{1}{\lambda_{1}^{2} \cdots \lambda_{p}^{2}}\right\rangle_{\rho_{\beta}}
$$

In terms of the integration variables (9), the sum rules become

$$
S_{p}=\left(\begin{array}{c}
n \\
p
\end{array}\right)\left(\frac{\beta n}{2 \sigma^{2}}\right)^{p} \frac{\left\langle\mu_{1} \cdots \mu_{n-p}\right\rangle_{\rho_{\beta, a-1}}}{\left\langle\mu_{1} \cdots \mu_{n}\right\rangle_{\rho_{\beta, a-1}}} .
$$

In order to make contact with Selberg's integral, the new average is with respect to $\rho_{\beta, a-1}$. Application of (20) yields

$$
S_{p}=\frac{1}{\prod_{k=0}^{p-1}\left(\beta+\beta \nu+2\left(N_{f}-1\right)+\beta k\right)}\left(\begin{array}{c}
n \\
p
\end{array}\right)\left(\beta n \Sigma^{2}\right)^{p} .
$$

Remarkably, for $N_{f}=1$ the variable $\beta$ drops out of the equation and the sum rule simplifies to

$$
S_{p}=\frac{\nu !}{(p+\nu) !}\left(\begin{array}{c}
n \\
p
\end{array}\right)\left(n \Sigma^{2}\right)^{p}
$$

This result constitutes the resolution of the paradox posed in the introduction. In fact,

we have constructed a one-parameter family of spectra which all give rise to the same one-flavor sum rules. Using that the total number of modes is $N=2 n$ the large $n$ limit of this sum rule is given by

$$
S_{p} \sim \frac{\nu !(N \Sigma)^{2 p}}{p !(p+\nu) ! 2^{2 p}}
$$

which coincides with a result obtained in [12, [] on general grounds involving the anomalous and chiral structure of QCD. For an arbitrary number of flavors, the large $n$ limit of (24) is given by

$$
S_{p}=\frac{\left(N^{2} \Sigma^{2}\right)^{p}}{2^{2 p} p !} \frac{\Gamma\left(\nu+1+2\left(N_{f}-1\right) / \beta\right)}{\Gamma\left(\nu+p+1+2\left(N_{f}-1\right) / \beta\right)},
$$

which again agrees with previous work [1].

The most general sum rule for $N_{f}=2$ is given by

$$
S_{p q}=\left\langle\sum_{\text {all } \lambda_{i} \text { different }} \frac{1}{\lambda_{m_{1}}^{4} \cdots \lambda_{m_{p}}^{4} \lambda_{n_{1}}^{2} \cdots \lambda_{n_{q}}^{2}}\right\rangle .
$$


These sum rules are also well-defined for any number of flavors $N_{f}>2$, and for less than two flavors only under certain conditions on $\nu$ and $\beta$. Therefore, we will evaluate the sum (27) for an arbitrary value of $N_{f}$, under the assumption that the integral is convergent. To evaluate the integrals we change variables as in the case of $N_{f}=1$. Exploiting the symmetry of the integration variables we obtain

$$
S_{p q}=N_{p q} \frac{\left\langle\prod_{k=1}^{n-p-q} \mu_{k}^{2} \prod_{l=n-p-q+1}^{n-p} \mu_{k}\right\rangle_{\rho_{\beta, a-2}}}{\left\langle\prod_{k=1}^{n} \mu_{k}^{2}\right\rangle_{\rho_{\beta, a-2}}}\left(\frac{\beta n \Sigma^{2}}{2}\right)^{q+2 p},
$$

where the total number of terms in (27) is denoted by

$$
N_{p q}=\frac{n !}{p ! q !(n-p-q) !}
$$

The integrals in (28) follow immediately from the recursion (21). The result can be expressed in terms of $\Gamma$-functions:

$$
S_{p q}=N_{p q} \frac{\Gamma\left(\alpha+\frac{2}{\beta}\right) \Gamma(\alpha+p) \Gamma\left(\alpha+\frac{2}{\beta}+p+n\right)}{\Gamma\left(\alpha+\frac{2}{\beta}+q+2 p\right) \Gamma(\alpha) \Gamma\left(\alpha+\frac{2}{\beta}+n\right)}\left(n^{2} \Sigma^{2}\right)^{q+2 p},
$$

where

$$
\alpha=\nu+1+\frac{2\left(N_{f}-2\right)}{\beta} .
$$

We observe that for $p=0$ these sum-rules reduce to the case $N_{f}=1$. For $p \neq 0$ and $N_{f}=1$ the $\beta$-dependence does not drop form (30). This is consistent with the fact that in this case the sum rules cannot be obtained from a chiral expansion of the partition function. For $N_{f} \neq 1$ the result depends on $\beta$. The thermodynamic limit of the sum-rules can be obtained with the help of Stirling's formula. This yields

$$
S_{p q} \sim \frac{\left(N^{2} \Sigma^{2}\right)^{q+2 p}}{4^{q+2 p} p ! q !} \frac{\Gamma\left(\alpha+\frac{2}{\beta}\right) \Gamma(\alpha)}{\Gamma\left(\alpha+\frac{2}{\beta}+q+2 p\right) \Gamma(\alpha+p)} .
$$

Two cases that have been considered before in [1], namely $\beta=2, p=1, q=0$ and $\beta=2$, $p=0, q=2$, are reproduced.

\section{Discussion}

In conclusion, we have evaluated all sum rules that follow from a chiral expansion of the partition function for one and two flavors. Each sum rule has been calculated for 
an arbitrary number of flavors and in a sector with a given topological charge. For one flavor we have found that all sum rules that can be derived from the effective low-energy partition function coincide for each of the three universality classes. This in spite of the fact that the spectral correlations are different. This resolves the paradox posed in the introduction of this letter. For two or more flavors, both the effective theory and the Leutwyler-Smilga sum rules are different for different values of $\beta$. However, we expect that also in this case the spectrum is not determined uniquely determined by the effective low-energy theory.

To see the connection between the spectral density near zero virtuality and the lowenergy effective theory, consider the partition function

$$
Z_{\nu}^{k}(m, z)=\left\langle\prod_{\lambda_{n}>0}\left(\lambda_{n}^{2}+z^{2}\right)^{k} \prod_{f=1}^{N_{f}} \prod_{\lambda_{n}>0}\left(\lambda_{n}^{2}+m_{f}^{2}\right) m_{f}^{\nu}\right\rangle_{S_{\nu}(A)} .
$$

The introduction of $k$ replicated flavors allows us to extract information on the spectral density. For $\nu=0$, for example, we have the relation

$$
2 \pi \rho(i z)=\lim _{k \rightarrow 0} \frac{1}{k} \frac{d}{d z} Z_{\beta, \nu=0}^{k}(m, z)
$$

To perform the $k \rightarrow 0$ limit of the effective partition function, we first evaluate it for any integer value of $k$ and take the limit after analytical continuation. This implies that in order to obtain the full spectral density for $N_{f}$ flavors, we do not only need the effective theory for $N_{f}$ flavors, but for any larger number of flavors (with a different mass) as well. Since the effective partition function for an arbitrary number of flavors with equal masses is known [1], this program may be feasible.

On the other hand, using techniques described in [11, 2] [13, the spectral density near zero virtuality may be related to an effective theory based on graded cosets. At the moment it is not clear which approach will be most successful.

\section{Acknowledgements}

The reported work was partially supported by the US DOE grant DE-FG-88ER40388. I would like to thank A. Smilga for pointing out the special status of $N_{f}=1$. 


\section{References}

[1] H. Leutwyler and A. Smilga, Phys. Rev. D46 (1992) 5607.

[2] E. Shuryak and J. Verbaarschot, Nucl. Phys. A560 (1993) (306).

[3] J.Verbaarschot, The spectrum of the Dirac operator and chiral random matrix theory: the threefold way, Stony Brook preprint SUNY-NTG-94/1 (1994).

[4] J. Verbaarschot and I. Zahed, Phys. Rev. Lett. 70 (1993) 3852.

[5] S. Dimopoulos, Nucl. Phys. B168 (1980) 69; M. Peskin, Nucl. Phys. B175 (1980) 197; M. Vysotskii, Y. Kogan and M. Shifman, Sov. J. Nucl. Phys. 42 (1985) 318; D. Diakonov and V. Petrov, in 'Quark Cluster Dynamics', Proceeding of the 99th WE-Heraeus Seminar, Bad Honnef, 1992 (eds. K. Goeke et al.), Springer 1993.

[6] F.J. Dyson, J. Math. Phys. 6 (1962) 1199.

[7] A. Smilga, private communication.

[8] A. Selberg, Norsk Mat. Tid. 26 (1944) 71.

[9] K. Aomoto, SIAM J. Math. Anal. 18 (1987) 545.

[10] M. Mehta, Random Matrices, Academic Press, San Diego, 1991.

[11] J. Verbaarschot, Chiral random matrix theory and the spectrum of the Dirac operator near zero virtuality, Acta Pol. Phys. (1994) (in press).

[12] A.Smilga, Phys. Rev. D46 (1992) 5607.

[13] J. Verbaarschot, H. Weidenmüller, and M. Zirnbauer, Phys. Rep. 129 (1985) 367.

[14] J. Verbaarschot, The spectrum of the Dirac operator near zero virtuality for $N_{c}=2$, Stony Brook preprint NTG-94/2 